\title{
Neurocognitive changes in pituitary adenoma patients after Gamma Knife radiosurgery
}

\author{
Alana Tooze, PhD, ${ }^{1}$ and Jason P. Sheehan, MD, PhD² \\ 1Sussex Rehabilitation Centre, Princess Royal Hospital, Haywards Heath, Sussex, United Kingdom; and 2Department of \\ Neurological Surgery, University of Virginia, Charlottesville, Virginia
}

OBJECTIVE Pituitary adenomas and the treatment required for the underlying neuropathology have frequently been associated with cognitive dysfunction. However, the mechanisms for these impairments remain the subject of much debate. The authors evaluated cognitive outcomes in patients treated with or without Gamma Knife radiosurgery (GKRS) for an underlying pituitary adenoma.

METHODS This was a retrospective, institutional review board-approved, single-institution study. A total of 51 patients (23 male, 28 female) treated for pituitary adenoma were included in this neurocognitive study. Twenty-one patients underwent GKRS following transsphenoidal surgery, 22 patients were treated with transsphenoidal surgery alone, and eight patients were conservatively managed or were treated with medical management alone. Comparisons using psychometric tests of general intellectual abilities, memory, and executive functions were made between the treatment groups, between male and female patients, and between patients with Cushing's disease and those with nonfunctioning adenoma (NFA).

RESULTS The entire patient sample, the NFA group, and the GKRS group scored significantly below expected on measures of both immediate and delayed memory, particularly for visually presented information ( $p \leq 0.05)$; however, there were no significant differences between the patients with Cushing's disease and those with NFA ( $t \leq 0.56, p \geq$ 0.52). In those who underwent GKRS, memory scores were not significantly different from those in the patients who did not undergo GKRS ( $t \leq 1.32, p \geq 0.19)$. Male patients across the sample were more likely to demonstrate impairments in both immediate memory $(t=-3.41, p=0.003)$ and delayed memory $(t=-3.80, p=0.001)$ than were female patients $(t \leq$ $1.09, p \geq 0.29$ ). There were no impairments on measures of general intellectual functioning or executive functions in any patient group. The potential contributions of tumor size and hormone levels are discussed.

CONCLUSIONS Overall, pituitary adenoma patients demonstrated relative impairment in anterograde memory. However, GKRS did not lead to adverse effects for immediate or delayed memory in pituitary adenoma patients. Cognitive assessment of pituitary adenoma patients is important in their longitudinal care.

https://thejns.org/doi/abs/10.3171/2018.7.GKS181595

KEYWORDS cognitive function; Cushing's; nonfunctioning adenoma; memory; stereotactic radiosurgery

$\mathrm{P}$ ITUITARY adenomas and the treatment required for the underlying neuropathology have frequently been associated with cognitive dysfunction. However, the nature of and the mechanisms for these impairments remain the subject of much debate. Surgery and ionizing radiation using either conventional radiotherapy or radio- surgery such as Gamma Knife radiosurgery (GKRS) are the treatments of choice for pituitary adenomas. The administration of GKRS in the post-resection period is highly effective in achieving tumor control of nonfunctioning adenomas (NFAs). $5,7,23$

However, pituitary adenoma treatments are not without

ABBREVIATIONS ACTH = adrenocorticotropic hormone; AMI = Auditory Memory Index; D-KEFS = Delis-Kaplan Executive Function System; DMI = Delayed Memory Index; FSIQ = Full Scale Intelligence Quotient; GH = growth hormone; GKRS = Gamma Knife radiosurgery; IMI = Immediate Memory Index; NFA = nonfunctioning adenoma; SHBG = sex hormone-binding globulin; T4 = thyroxine; TSH = thyroid-stimulating hormone; VMI = Visual Memory Index; VWMI = Visual Working Memory Index; WAISIV = Wechsler Adult Intelligence Scale-Fourth Edition; WMS-IV = Wechsler Memory Scale-Fourth Edition.

SUBMITTED June 1, 2018. ACCEPTED July 26, 2018.

INCLUDE WHEN CITING DOI: 10.3171/2018.7.GKS181595. 
complications. Patients treated for pituitary adenoma have reported memory impairments, ${ }^{19,38}$ and several studies have found deficits in various domains of memory and executive function. In a recent systematic review, Alsumali et al. ${ }^{1}$ compared nine studies of cognition following transsphenoidal surgery for pituitary tumors. Six of the eight cross-sectional studies found postoperative impairments in verbal and nonverbal memory; however, two studies found no decline or even improvement in memory postsurgery. There was even greater variance between the studies in other assessed areas of cognition, with half of the studies showing postoperative impairment in attention or executive functioning, while the other half did not. Overall, Alsumali et al. found that negative changes in verbal memory were the most consistent findings postsurgery. In contrast, other studies have consistently found that general intellectual functioning is unaffected. . $^{15,26,33,34}$

Most studies examining the cognitive effects of radiotherapy are based on conventional three-field radiotherapy, although more recent studies of cognition following GKRS have begun to emerge. Many studies of conventional radiotherapy have found no differences between patients treated with and those treated without irradiation, $8,14,15,26$ although some differences have occasionally been found on measures of executive ability ${ }^{24}$ and memory. ${ }^{18}$ Several reasons for these differences have been noted, but again these are inconsistently noted. One study found that younger patients had better outcomes than older patients after pituitary surgery, ${ }^{22}$ while others have not reported a difference. , $^{8,24,26,27,34,35}$ Another study found that presurgery tumor size is negatively correlated with postsurgery outcome, ${ }^{28}$ whereas other studies have not. ${ }^{16,35}$ Finally, Tooze ${ }^{33}$ found a sex difference, with men performing significantly worse than women on tests of immediate memory across a cohort of patients treated for NFA, regardless of treatment group. Interestingly, a previous study ${ }^{14}$ added patient sex as a covariate in its analyses, suggesting a difference between men and women in their sample, although this was not independently reported. Other studies have not reported whether they found a sex difference. ${ }^{24,26,27,34,35}$

Studies on the cognitive effects of GKRS have started to appear. A preliminary pilot study of the effects of GKRS on cognition found that while patients treated for pituitary adenoma demonstrated memory impairment, there were no cognitive differences between those treated with and those treated without GKRS. ${ }^{34}$ A longitudinal study screened six different areas of cognition and functioning before stereotactic irradiation and for an average of 24 months thereafter. ${ }^{9}$ While there was evidence of some deterioration for individual patients, the patient group as a whole did not show evidence of cognitive deterioration following irradiation and even showed improvement on a measure of executive function. While these initial studies are promising, the first was a pilot study conducted on a small sample of 14 patients $^{34}$ and the second study used only one computer-based test of each cognitive function, which would be considered more of a cognitive screen than a full analysis of these cognitive abilities. ${ }^{6}$

In the current study we aim to expand on our preliminary pilot study and deliver the first full-powered cross- sectional comparison of the cognitive abilities of patients treated with or without GKRS for pituitary adenoma, using the current gold-standard psychometrics. In this study we use a measure of relative impairment (i.e., the predictive discrepancy) in assessments of memory, which does not show bias if a person's general intellectual abilities deviate from those of their age-matched peers. This should allow the most sensitive comparisons of these treatment groups. Our null hypothesis is that there will be no difference between treatment groups or patient groups on cognitive measures, and our alternative hypothesis is that patients treated with GKRS will have poorer outcomes on cognitive measures than those treated without the use of GKRS. Furthermore, our alternative hypothesis is that patients treated for NFA will perform better on tests of cognition than patients with hormone-producing pituitary tumors.

\section{Methods}

This is a retrospective cross-sectional comparison of patients treated for pituitary adenoma using measures of general intellectual ability, memory, and executive functioning. The study was approved by the University of Virginia institutional review board. Patients had been seen in the neuro-endocrine and neurosurgery clinics at the University of Virginia Medical Center. During the period of 2014 to 2016, 14 patients were treated for Cushing's disease, 7 for acromegaly, 4 for medically refractory prolactinoma, and 26 for NFAs. Eight patients in the non-GKRS group were conservatively managed (i.e., 6 patients with NFA had no radiosurgical, radiation, medical, or resective treatment) or were treated with medication alone (2 patients with prolactinoma). All other patients underwent surgery. All patients were diagnosed with a pituitary adenoma after the age of 18 years. Patients were treated with resection or radiosurgery a minimum of 6 months before testing. Patients were excluded from the study if they had a history of stroke, known neurological impairment, or diabetes mellitus or were pregnant or breastfeeding.

The radiosurgical technique utilized at our institution has been previously described. ${ }^{34}$ During the study period, patients were treated with GKRS in a single fraction. Multi-isocenter dose plans were used. Patients with NFAs received a mean margin dose of $17 \mathrm{~Gy}$, and those with functioning adenomas such as Cushing's disease received a mean margin dose of $25 \mathrm{~Gy}$. In patients with NFAs and functioning adenomas, the ipsilateral maximal doses to the hippocampus were approximately 4-11 Gy and 8-14 Gy, respectively. In a study by our group in which we looked at the radiation dose to the anterior temporal lobe structures of radiosurgically treated patients, the mean dose and 50\% volume dose to the anterior temporal lobe structures were $2.6 \mathrm{~Gy}$ and $0.6 \mathrm{~Gy}$, respectively. ${ }^{3}$

To evaluate preoperative endocrine function, patients underwent testing of 24-hour urine free cortisol (UFC) for Cushing's disease, free thyroxine (T4), thyroid-stimulating hormone (TSH), prolactin, follicle-stimulating hormone, luteinizing hormone, growth hormone $(\mathrm{GH})$, insulin-like growth factor (IGF)-1, serum cortisol, adrenocorticotropic hormone $(\mathrm{ACTH})$, testosterone in men, estradiol and 
menstrual history in women, and a basic metabolic panel. For postintervention endocrine follow-up, patients had endocrine testing specific to their functioning adenoma type to assess response to treatment typically at 6-month intervals for the 1st year and yearly thereafter. At the discretion of the treating endocrinologist and if clinically indicated, additional laboratory testing was performed. Adrenocorticotropic hormone deficiency was defined as a subnormal serum cortisol level with concomitant low ACTH levels. Serum cortisol levels were corrected to the reference range based on the timing of the testing. A TSH deficiency was defined as low free T4 levels with normal or decreased TSH. Gonadotropin deficiency was defined as low plasma testosterone with nonelevated gonadotropin levels in men, low plasma estradiol with low or normal gonadotropins in premenopausal women, or a lack of increased gonadotropins in postmenopausal women. A GH deficiency was defined as a low response of $\mathrm{GH}$ to insulin tolerance test (peak $\mathrm{GH}<5 \mathrm{ng} / \mathrm{ml}$ ). If the endocrine testing was performed at an outside laboratory, we relied on the normal reference ranges provided from the testing laboratory to determine the presence of a pituitary deficiency for each particular hormone tested.

\section{Neurocognitive Procedure}

Patients completed the Wechsler Adult Intelligence Scale-Fourth Edition (WAIS-IV), the Wechsler Memory Scale-Fourth Edition (WMS-IV), and the Delis-Kaplan Executive Function System (D-KEFS), with rest breaks as needed. Forty-two patients completed the adult version of the WMS-IV, while 9 patients over the age of 69 years completed the older adult version of the WMS-IV, which does not provide the Visual Working Memory Index (VWMI).

\section{Neurocognitive Measures}

The D-KEFS measures various executive functions such as verbal fluency, inhibition, hypothesis testing, strategy use, and problem solving. The WAIS-IV measures both verbal and performance intelligence with a range of tasks that measure skills such as perceptual abilities and abstract reasoning. The WMS-IV is a battery of measures assessing anterograde memory. Participants are asked to recall either visually or verbally presented stimuli directly after each one is presented and then once more between 20 and 30 minutes later, thus measuring both immediate and delayed recall. The WAIS-IV was originally trialed on 2200 neurologically intact individuals to collect the normative test data. These individuals were between 16 and 90 years of age. The WAIS-IV was co-normed with the WMS-IV.

The WMS-IV was originally normed on 1400 of the same individuals who took the WAIS-IV, meaning the correlations between the WAIS-IV and WMS-IV could be evaluated to determine their relationships. The relatively high correlations between general intellectual abilities and memory allow the use of discrepancy analysis, wherein each person's IQ score provides an estimate of their memory abilities. ${ }^{36}$ Each individual's obtained memory scores can then be compared to the estimated scores predicted by their Full Scale Intelligence Quotient (FSIQ). While there is not a completely linear relationship between FSIQ and memory, FSIQ is a better predictor of memory abilities than any other index. When used in group studies, this method reduces within-group variance because each individual is compared to their theoretical selves, allowing comparison of each group to their expected scores, not just to other groups.

The WAIS-IV and WMS-IV were counter-balanced so an equal number of patients in each group started the testing session with each test. This prevented fatigue from affecting the results of one test more than the other. The results of the D-KEFS were not compared to the other tests, so the D-KEFS was administered last. This ensured the minimum amount of data was lost if a participant chose to leave the research protocol early. The psychometrics took a total of 4 hours to complete. The resulting data were analyzed using SPSS version 23 (IBM SPSS Statistics for Windows, version 23.0, IBM Corp.). Statistical significance was deemed to have been achieved with a $p$ value of less than 0.05 .

Absolute impairment measures an individual's abilities in comparison to those of their age-matched peers. In contrast, relative impairment measures the discrepancy between each individual's memory function and that same individual's general intellectual functioning. Thus, the FSIQ of each patient was used to predict their memory scores using the following formula: achieved memory score - predicted memory score $=$ discrepancy score ${ }^{36}$ This is called the "predicted difference method." It represents a more sensitive assessment of potential memory dysfunction. A negative discrepancy score indicates performance that is worse than expected and may indicate an acquired memory deficit. By predicting each individual's memory scores from their own general intellectual abilities, differences between groups that are a result of a baseline difference in general ability will have a reduced effect on the study results, by decreasing between-group variance.

To test for relative impairment within each group, the difference between each group's expected scores and their observed memory scores was compared to a score of 0 (no difference between observed and predicted scores) using a one-sample t-test.

The data were analyzed in four ways. Firstly, the whole group was analyzed to identify whether or not there was a consistent pattern of cognitive deficits across diagnoses and treatments. Secondly, patients with NFA were compared to patients with Cushing's disease to explore any potential additional impairment shown by the patients with Cushing's disease possibly attributable to their raised cortisol levels. Thirdly, patients treated with GKRS were compared to patients who were treated without the use of any form of radiosurgery or radiotherapy to examine any additional impairment in the patients treated with GKRS. Finally, where differences were found, we conducted a post hoc comparison between male and female patients.

\section{Results}

The demographic and treatment characteristics of the participants are shown in Table 1. The characteristics were 
TABLE 1. Demographic and treatment characteristics of study subjects

\begin{tabular}{lccc}
\hline \multicolumn{1}{c}{ Parameter } & No GKRS & GKRS & $p$ Value \\
\hline No. of patients & $30(12 \mathrm{NFA} / 10 \mathrm{C} / 5 \mathrm{~A} / 3 \mathrm{P})$ & $21(14 \mathrm{NFA} / 4 \mathrm{C} / 2 \mathrm{~A} / 1 \mathrm{P})$ & $\mathrm{NA}$ \\
\hline Sex & $11 \mathrm{M} / 19 \mathrm{~F}$ & $12 \mathrm{M} / 9 \mathrm{~F}$ & $\mathrm{NA}$ \\
\hline Age in yrs & $56.2 \pm 13.9$ & $55.8 \pm 12.2$ & 0.90 \\
\hline Tumor size at start of treatment in $\mathrm{cm}^{3}$ & $1.54 \pm 3.78$ & $1.76 \pm 2.03$ & \\
\hline Tumor size at cognitive testing in $\mathrm{cm}^{3}$ & $0.49 \pm 1.33$ & $0.43 \pm 0.86$ & \\
\hline Time since resection in mos & $70.4 \pm 69.2$ & $86.8 \pm 38.0$ & 0.82 \\
\hline Time since SRS in mos & $\mathrm{NA}$ & $53.3 \pm 35.0$ & 0.43 \\
\hline
\end{tabular}

$\mathrm{A}=$ acromegaly; $\mathrm{C}=$ Cushing's disease NA = not applicable; NFA = nonfunctioning adenoma; $\mathrm{P}=$ prolactinoma; $\mathrm{SRS}=$ stereotactic radiosurgery.

Data are expressed as the mean \pm standard deviation, unless indicated otherwise.

compared using independent samples t-tests. The groups did not differ significantly on any characteristic. No differences for age $(\mathrm{t}=0.13, \mathrm{p} \geq 0.90)$ or the male/female ratio ( $\mathrm{p}=0.15$, chi-square test) were found between the groups.

\section{Intelligence}

The mean FSIQ and index scores for each subgroup are shown in Table 2 . There were no significant differences between patients treated with or without GKRS $(p \geq 0.07)$ or between the patients with NFA and those with Cushing's disease $(p \geq 0.09)$. A one-sample t-test demonstrated that the mean FSIQ for the whole sample was significantly higher than the general population mean score of $100(\mathrm{t}=$ $3.37, \mathrm{p}=0.001$ ).

\section{Memory}

The memory scores obtained by each subgroup are shown in Table 3. The discrepancies between each patient's predicted memory scores and their achieved memory scores were compared to 0 (no discrepancy) and are shown in Table 4.

The Visual Memory Index (VMI) discrepancy scores $(\mathrm{t}=-3.92, \mathrm{p}<0.001)$, Immediate Memory Index (IMI) discrepancy scores $(\mathrm{t}=-2.95, \mathrm{p}=0.005)$, and Delayed Memory Index (DMI) discrepancy scores $(t=-2.57, p=$ 0.01 ) for the entire sample were significantly lower than the scores predicted by the patients' FSIQ. Therefore, the whole patient sample shows a consistent pattern of relative memory impairment. Both patients with NFA and patients treated with GKRS performed significantly worse than expected on the VMI, the IMI, and the DMI, and the patients treated with GKRS also scored below expected on the Auditory Memory Index (AMI). Patients treated without GKRS only scored below expected on the VMI. Every patient group scored as well as expected based on their FSIQ on the Visual Working Memory Index (VWMI). However, there were no significant differences in the memory scores between the patients with NFA and those with Cushing's disease ( $t \leq 0.56, p \geq 0.52)$, nor between the patients treated with and those treated without GKRS ( $\mathrm{t} \leq$ $1.32, p \geq 0.19)$. Interestingly, there were also no differences in the memory or discrepancy scores between the eight patients who had not undergone surgery and the patients who had undergone surgery $(t \leq 1.35, p \geq 0.19)$ or surgery and GKRS ( $t \leq 1.346, p \geq 0.16)$, except for the VWMI discrepancy scores, on which the surgically treated patients tended toward better performance than those treated without surgery or GKRS $(\mathrm{t}=1.91, \mathrm{p}=0.07)$.

Post hoc analyses were conducted to explore other factors that could account for the significant discrepancies between predicted and achieved memory scores seen in some patients. There was no correlation between tumor size, either at the start of treatment $(r \leq 0.17, p \geq 0.25)$ or at the point of cognitive testing ( $\mathrm{r} \leq 0.18, \mathrm{p} \geq 0.22)$, and any of the memory scores for the whole sample or for the NFA group, which contained the most patients who had undergone surgical intervention (20/26 patients). However, a significant difference was found between the male and female patients on the AMI discrepancy score, with men

TABLE 2. General intellectual functioning of the sample

\begin{tabular}{cccccc}
\hline Parameter & Whole Sample & GKRS & No GKRS & Cushing's & NFA \\
\hline No. of patients & 51 & 21 & 30 & 14 & 26 \\
\hline FSIQ & $107.1(14.8)$ & $109.4(13.1)$ & $105.5(15.9)$ & $109.6(15.1)$ & $107.3(15.1)$ \\
\hline Index & & & & & \\
\hline VCI & $110.7(16.3)$ & $110.5(15.4)$ & $110.8(17.1)$ & $117.1(17.9)$ & $107.8(14.7)$ \\
\hline PRI & $104.8(15.1)$ & $106.2(12.7)$ & $103.8(16.7)$ & $104.7(15.3)$ & $107.0(15.7)$ \\
\hline WMI & $103.8(15.2)$ & $108.0(16.6)$ & $101.0(13.9)$ & $104.5(13.6)$ & $105.4(17.0)$ \\
\hline PSI & $101.7(12.1)$ & $105.4(9.9)$ & $99.2(12.9)$ & $102.6(12.0)$ & $101.6(10.8)$ \\
\hline
\end{tabular}

FSIQ = Full Scale Intelligence Quotient; PRI = Perceptual Reasoning Index; PSI = Processing Speed Index; VCI = Verbal Comprehension Index; $\mathrm{WMI}=$ Working Memory Index.

Mean scores are shown with standard deviations in brackets. 
TABLE 3. WMS-IV scores achieved by each group

\begin{tabular}{lccccc}
\hline Parameter & Whole Sample & GKRS & No GKRS & Cushing's & NFA \\
\hline No. of patients & 51 & 21 & 30 & 14 & 26 \\
\hline Index & & & & & \\
\hline AMI & $101.6(15.9)$ & $99.7(12.5)$ & $103.0(18.1)$ & $105.9(20.7)$ & $102.5(10.6)$ \\
\hline VMI & $100.3(13.3)$ & $101.1(13.1)$ & $99.7(13.6)$ & $102.4(11.9)$ & $99.8(12.7)$ \\
\hline VWMI & $104.8(12.0)(n=42)$ & $107.8(11.5)(n=17)$ & $102.8(12.1)(n=25)$ & $105.9(10.5)(n=13)$ & $105.9(14.3)(n=19)$ \\
\hline IMI & $101.1(15.2)$ & $99.9(13.0)$ & $101.9(16.7)$ & $104.9(18.7)$ & $101.3(12.2)$ \\
\hline DMI & $101.5(15.1)$ & $101.2(13.0)$ & $101.8(16.6)$ & $104.9(18.1)$ & $102.0(12.0)$ \\
\hline
\end{tabular}

AMI = Auditory Memory Index; DMI = Delayed Memory Index; IMI = Immediate Memory Index; $n=$ number of patients; VMI = Visual Memory Index; VWMI = Visual Working Memory Index.

Mean scores are shown with standard deviations in brackets.

demonstrating greater discrepancies between their predicted and achieved scores than women $(\mathrm{t}=2.51, \mathrm{p}=0.02)$. There was also a trend toward significance for the DMI discrepancy score $(\mathrm{t}=1.96, \mathrm{p}=0.06)$, with women achieving delayed memory scores that were more commensurate with their predicted scores than men.

Finally, potential correlations between the memory discrepancy scores and hormone levels were investigated. Free T4 correlated with the VWMI discrepancy score. Patients with higher free T4 levels were more likely to achieve VWMI scores that were commensurate with the scores they were expected to attain, whereas patients with lower free T4 levels were more likely to achieve VWMI scores that were lower than expected $(r=0.31, p=0.05)$.
However, the more notable correlation was between sex hormone-binding globulin (SHBG; measured in 20 male patients) and the memory index scores. Interestingly, SHBG strongly negatively correlated with the AMI $(\mathrm{r}=$ $-0.49, \mathrm{p}=0.03)$, the VMI $(\mathrm{r}=-0.48, \mathrm{p}=0.03)$, the IMI $(\mathrm{r}=$ $-0.51, p=0.02)$, and the DMI $(r=-0.52, p=0.02)$. However, SHBG did not correlate with the memory discrepancy scores $(p \geq 0.17)$. This correlation was not replicated with free testosterone $(r \leq-0.13, p \geq 0.56)$ or testosterone per se $(r \leq-0.31, p \geq 0.16)$. In order to elucidate why SHBG could be negatively correlated with the memory index scores, but not the discrepancy scores, a further correlation between FSIQ and SHBG was tested, which showed a trend toward a significant negative correlation $(r=-0.41, p=0.08)$.

TABLE 4. Memory scores predicted for each group and the discrepancy from the achieved scores

\begin{tabular}{|c|c|c|c|c|c|}
\hline Parameter & Whole Sample & GKRS & No GKRS & Cushing's & NFA \\
\hline No. of patients (no. of M/F) & $51(23 \mathrm{M} / 23 \mathrm{~F})$ & $21(12 \mathrm{M} / 9 \mathrm{~F})$ & $30(11 \mathrm{M} / 19 \mathrm{~F})$ & $14(3 \mathrm{M} / 11 \mathrm{~F})$ & $26(16 \mathrm{M} / 10 \mathrm{~F})$ \\
\hline \multicolumn{6}{|l|}{ Index } \\
\hline \multicolumn{6}{|l|}{ AMI } \\
\hline Predicted mean & 104.8 & 105.1 & 104.5 & 106.6 & 104.6 \\
\hline Discrepancy & $-3.2(13.4)$ & $-5.7(11.8)$ & $-1.6(14.4)$ & $-0.6(17.0)$ & $-2.2(9.6)$ \\
\hline$p(t) \neq 0$ & 0.10 & 0.05 & 0.56 & 0.89 & 0.27 \\
\hline \multicolumn{6}{|l|}{ VMI } \\
\hline Predicted mean & 105.9 & 106.7 & 105.3 & 107.9 & 106.0 \\
\hline Discrepancy & $-5.7(10.1)$ & $-5.8(7.5)$ & $-5.6(11.7)$ & $-5.5(10.9)$ & $-6.5(9.1)$ \\
\hline$p(t) \neq 0$ & $<0.001$ & 0.003 & 0.02 & 0.09 & 0.002 \\
\hline \multicolumn{6}{|l|}{ VWMI } \\
\hline Predicted mean & 106.1 & 107.1 & 105.4 & 109.0 & 105.1 \\
\hline Discrepancy & $-2.3(10.8)$ & $-2.1(12.4)$ & $-2.5(9.9)$ & $-3.2(11.4)$ & $-1.9(11.1)$ \\
\hline$p(t) \neq 0$ & 0.17 & 0.50 & 0.22 & 0.34 & 0.48 \\
\hline \multicolumn{6}{|l|}{ IMI } \\
\hline Predicted mean & 106.0 & 106.5 & 105.7 & 108.2 & 105.9 \\
\hline Discrepancy & $-5.1(12.1)$ & $-7.0(10.8)$ & $-3.8(13.0)$ & $-3.3(15.5)$ & $-4.8(9.9)$ \\
\hline$p(t) \neq 0$ & 0.005 & 0.01 & 0.12 & 0.44 & 0.03 \\
\hline \multicolumn{6}{|l|}{ DMI } \\
\hline Predicted mean & 105.7 & 106.3 & 105.2 & 107.9 & 105.7 \\
\hline Discrepancy & $-4.2(11.6)$ & $-5.4(9.1)$ & $-3.5(13.1)$ & $-2.9(15.1)$ & $-3.8(9.1)$ \\
\hline$p(t) \neq 0$ & 0.01 & 0.02 & 0.17 & 0.50 & 0.05 \\
\hline
\end{tabular}

Mean scores are shown with standard deviations in brackets. Boldface type indicates statistical significance. 


\section{Executive Functions}

A one-sample t-test showed that the whole sample's scores were either commensurate with those of the general population or significantly better than those of the general population ( $\mathrm{t} \geq 1.43, \mathrm{p} \leq 0.16$ ), and a similar pattern of results was found across all subgroups. Unsurprisingly, the executive functioning scores highly correlated with FSIQ $(r \geq 0.46, p \leq 0.001)$, except for the Tower test, which only tended toward significance $(r=2.56, p=0.07)$. There were no significant differences in the executive functioning scores between the patients with Cushing's disease and those with NFA ( $\mathrm{t} \leq 1.42, \mathrm{p} \geq 0.17$ ), nor between the patients treated with and those treated without GKRS ( $\mathrm{t} \leq$ $1.29, \mathrm{p} \geq 0.20$ ).

\section{Discussion}

This study compared the cognitive outcome of patients treated with or without GKRS for pituitary adenoma and found no impairment in general intellectual functioning, anterograde memory, or executive abilities compared to the normative data from gold-standard cognitive tests. These results are consistent with the findings in our previous pilot study. ${ }^{34}$ We compared differences in neurocognitive function between appropriately powered cohorts treated with GKRS and those treated without GKRS for pituitary adenoma. The power analysis was based on our previous preliminary study. ${ }^{34}$ Thus, although we did not have baseline neurocognitive function testing before intervention, we did have sufficient cohort sizes to detect differences in neurocognitive function as a result of differences in treatment modalities.

When using the more sensitive "predicted difference method" (discrepancy score) to assess memory functioning, the patient group scored worse than would be expected, given their FSIQs, on measures of both immediate and delayed memory, with greater deficits found for visually presented information than for verbally presented information. Patients treated with GKRS scored significantly below expected on measures of both immediate and delayed memory, whereas patients treated without GKRS did not. It should be noted, however, that a direct comparison of these two treatment groups did not show a significant difference, suggesting that if there is further acquired memory impairment post-GKRS, these differences are likely to be subtle in most cases.

Further analysis of relative memory impairment showed that tumor size at the time of surgery or at the time of testing was not correlated with memory abilities. Interestingly, a sex difference was found within the entire sample, with men demonstrating greater levels of relative memory impairment than women. This is the second study to find a sex difference in relative memory performance. When assessing a cohort of patients treated for NFA in the United Kingdom, Tooze ${ }^{33}$ found that men were more likely to experience relative memory impairment than women, regardless of treatment type. Interestingly, patients treated for Cushing's disease did not perform significantly differently from the patients treated for NFA. They were more likely to achieve memory scores commensurate with their FSIQ. This result is counter to the current literature ${ }^{12,13,29,32}$ and may be a result of the lower number of men in the patient group with Cushing's disease.

Furthermore, when the correlation between memory scores and hormone levels was examined, SHBG negatively correlated with memory abilities. Due to a trend toward a further negative correlation between SHBG and FSIQ, the memory discrepancy scores were not correlated with SHBG. This means that higher SHGB is associated with lower bioavailability of testosterone, lower memory scores, and potentially lower general intellectual functioning. This correlation was not replicated with measures of testosterone.

Until recent years, few studies have examined the relationship between SHBG and cognition; instead, they more frequently explore the relationship between free testosterone and cognition, without reference to its bioavailability. However, studies that have examined the relationship between SHBG and cognition have found a fairly consistent negative relationship between memory and SHBG, both in large-scale population-based studies and smaller studies. ${ }^{2,7,11,31}$ The strongest correlations between SHBG and cognition are found in studies of dementia, where SHBG has been associated with an increased likelihood of developing Alzheimer's dementia and poorer cognitive scores in both men and women. 17,20,25,37 Levels of SHBG have been linked to changes in the brain in men as they age. ${ }^{10}$ However, while SHBG negatively correlated with all the main measures of memory in the present study, this result must be interpreted with caution. A correlation between SHBG and memory was not found in the previous United Kingdom study, despite the sex difference found (A. Tooze, personal communication, 2018), and so this finding will need to be replicated before any causality can be inferred. Finally, the positive correlation between free T4 and working memory has been found elsewhere and is thought to be related to the frontoparietal network. . $^{30,39}$

There are several potential reasons patients with pituitary adenoma can experience cognitive dysfunction; principally, these are mass effect of tumor, treatment, hormone dysfunction, and/or nonorganic reasons such as health anxiety. This study supports the hypothesis that hormone dysfunction is the principal cause of cognitive deficits found. We did not find a correlation between cognitive abilities and tumor size, either at diagnosis or at the time of assessment, thus refuting the hypothesis that mass effect is deleterious to cognition. Secondly, we did not find a difference between the small number of patients treated without surgery, those treated with surgery, and those treated with both surgery and GKRS, suggesting that treatment itself is not a primary cause of cognitive change, although the small number of patients treated without surgery means that this result must be handled with caution. Thirdly, given that all patients were at least 6 months posttreatment and that most were several years posttreatment, they would be expected to have had sufficient time to come to terms with their diagnosis and treatment. Thus, it would be unlikely that large numbers of these patients would demonstrate cognitive impairment due to health anxiety so long after diagnosis and treatment. Finally, the correlation between SHBG and memory abilities, potentially resulting in the sex difference found, would suggest hormone dys- 
function as the most likely reason for these results. These results suggest that, when counseling patients, clinicians should take concerns about cognition seriously, especially as there can be marked variability in acquired dysfunction within this patient group. However, clinicians should also feel confident in reassuring patients that treatment does not, on average, appear to significantly worsen any existing cognitive decline. Notably, this study highlights the importance of the endocrinologist within the clinical team.

Further studies assessing cognitive changes in this patient group are still needed. There are some flaws and omissions in the previous literature, potentially contributing to the variance in results reported. Differing control groups have been used across studies. Some have used a small control group of neurologically intact individuals, ${ }^{15,16,26,27}$ whereas others have compared their cohort to a group of patients with a different chronic condition. . $^{8,14,21}$ Few studies have compared patient groups to standardized test norms..$^{4,15,24}$ Most studies have used limited comparisons of absolute impairment (i.e., patient performance on tasks of memory). This method can be insensitive as within-group variation is likely to be substantial in comparison to the between-group variation attributable to treatment effects. The current study has highlighted the importance of using sensitive measures of relative impairment when assessing this patient group to capture potential acquired deficits, especially in patients with above-average intelligence. The higher premorbid cognitive baseline of these patients can mask subtle acquired deficits. Our study is cross-sectional, and future longitudinal studies performing cognitive testing before and after treatment would be advantageous. Analysis of potential sex differences and whether SHBG has a role in acquired deficits in this patient group would also be welcomed.

\section{Conclusions}

Functioning and nonfunctioning pituitary adenoma patients often demonstrate some degree of impairment in anterograde memory; however, GKRS did not lead to demonstrable effects regarding immediate or delayed memory for pituitary adenoma patients. The assessment of cognitive function of pituitary adenoma patients is important in their longitudinal care.

\section{Acknowledgments}

Drs. Vance and Hong, neuro-endocrinologists at the University of Virginia, assisted in patient recruitment. Ms. Johanna Loomba, Ms. Jennifer De Jong, and Ms. Amy Warren in the University of Virginia neuroclinical trials office assisted with trial coordination. Dr. Amitabh Gupta assisted with some data collection, and we are grateful for his help. Finally, grant support for this study was given to the University of Virginia from Elekta.

\section{References}

1. Alsumali A, Cote DJ, Regestein QR, Crocker E, Alzarea A, Zaidi HA, et al: The impact of transsphenoidal surgery on neurocognitive function: a systematic review. J Clin Neurosci 42:1-6, 2017

2. Barrett-Connor E, Goodman-Gruen D, Patay B: Endogenous sex hormones and cognitive function in older men. J Clin Endocrinol Metab 84:3681-3685, 1999
3. Basina BR, Olson C, Roy DK, Yen CP, Schlesinger D, Nagayama K, et al: Radiation dose and incidence of new metastasis in the anterior temporal lobe structures of radiosurgically treated patients. J Neurosurg 112:122-129, 2010

4. Baum HBA, Katznelson L, Sherman JC, Biller BMK, Hayden DL, Schoenfeld DA, et al: Effects of physiological growth hormone $(\mathrm{GH})$ therapy on cognition and quality of life in patients with adult-onset GH deficiency. J Clin Endocrinol Metab 83:3184-3189, 1998

5. Bir SC, Murray RD, Ambekar S, Bollam P, Nanda A: Clinical and radiologic outcome of Gamma Knife radiosurgery on nonfunctioning pituitary adenomas. J Neurol Surg B Skull Base 76:351-357, 2015

6. Blum WF, Shavrikova EP, Edwards DJ, Rosilio M, Hartman ML, Marín F, et al: Decreased quality of life in adult patients with growth hormone deficiency compared with general populations using the new, validated, self-weighted questionnaire, questions on life satisfaction hypopituitarism module. J Clin Endocrinol Metab 88:4158-4167, 2003

7. Bove RM, Gerweck AV, Mancuso SM, Bredella MA, Sherman JC, Miller KK: Association between adiposity and cognitive function in young men: Hormonal mechanisms. Obesity (Silver Spring) 24:954-961, 2016

8. Brummelman P, Elderson MF, Dullaart RPF, van den Bergh ACM, Timmer CA, van den Berg G, et al: Cognitive functioning in patients treated for nonfunctioning pituitary macroadenoma and the effects of pituitary radiotherapy. Clin Endocrinol (Oxf) 74:481-487, 2011

9. Burger H, Vernimmen FJAI, Dugmore D, Parkes JD, Balchin RM: Evaluation of the potential of inducing neurocognitive changes in patients receiving intracranial stereotactic irradiation for benign tumors: a preliminary study. J Radiosurg SBRT 3:1-11, 2014

10. Elbejjani M, Schreiner PJ, Siscovick DS, Sidney S, Lewis CE, Bryan NR, et al: Sex hormones and brain volumes in a longitudinal study of middle-aged men in the CARDIA study. Brain Behav 7:e00765, 2017

11. Fonda SJ, Bertrand R, O'Donnell A, Longcope C, McKinlay JB: Age, hormones, and cognitive functioning among middle-aged and elderly men: cross-sectional evidence from the Massachusetts Male Aging Study. J Gerontol A Biol Sci Med Sci 60:385-390, 2005

12. Forget H, Lacroix A, Bourdeau I, Cohen H: Long-term cognitive effects of glucocorticoid excess in Cushing's syndrome. Psychoneuroendocrinology 65:26-33, 2016

13. Forget H, Lacroix A, Somma M, Cohen H: Cognitive decline in patients with Cushing's syndrome. J Int Neuropsychol Soc 6:20-29, 2000

14. Grattan-Smith PJ, Morris JGL, Shores EA, Batchelor J, Sparks RS: Neuropsychological abnormalities in patients with pituitary tumours. Acta Neurol Scand 86:626-631, 1992

15. Guinan EM, Lowy C, Stanhope N, Lewis PDR, Kopelman MD: Cognitive effects of pituitary tumours and their treatments: two case studies and an investigation of 90 patients. $\mathbf{J}$ Neurol Neurosurg Psychiatry 65:870-876, 1998

16. Hendrix P, Griessenauer CJ, Hans E, Simgen A, Oertel J, Karbach J: Cognitive function surrounding resection of nonfunctioning pituitary adenomas with suprasellar extension: a prospective matched-control study. J Clin Neurosci 40:109-114, 2017

17. Hoskin EK, Tang MX, Manly JJ, Mayeux R: Elevated sexhormone binding globulin in elderly women with Alzheimer's disease. Neurobiol Aging 25:141-147, 2004

18. Lecumberri B, Estrada J, García-Uría J, Millán I, Pallardo LF, Caballero L, et al: Neurocognitive long-term impact of two-field conventional radiotherapy in adult patients with operated pituitary adenomas. Pituitary 18:782-795, 2015

19. McCord MW, Buatti JM, Fennell EM, Mendenhall WM, 
Marcus RB Jr, Rhoton AL, et al: Radiotherapy for pituitary adenoma: long-term outcome and sequelae. Int J Radiat Oncol Biol Phys 39:437-444, 1997

20. Muller M, Schupf N, Manly JJ, Mayeux R, Luchsinger JA: Sex hormone binding globulin and incident Alzheimer's disease in elderly men and women. Neurobiol Aging 31:17581765,2010

21. Müssig K, Besemer B, Saur R, Klingberg S, Häring HU, Gallwitz B, et al: Deteriorated executive functions in patients with successful surgery for pituitary adenomas compared with other chronically ill patients. J Int Neuropsychol Soc 17:369-375, 2011

22. Müssig K, Leyhe T, Besemer B, Saur R, Häring HU, Gallwitz $\mathrm{B}$, et al: Younger age is a good predictor of better executive function after surgery for pituitary adenoma in adults. J Int Neuropsychol Soc 15:803-806, 2009

23. Narayan V, Mohammed N, Bir SC, Savardekar AR, Patra DP, Bollam P, et al: Long-term outcome of nonfunctioning and hormonal active pituitary adenoma after Gamma Knife radiosurgery. World Neurosurg 114:e824-e832, 2018

24. Noad R, Narayanan KR, Howlett T, Lincoln NB, Page RCL: Evaluation of the effect of radiotherapy for pituitary tumours on cognitive function and quality of life. Clin Oncol (R Coll Radiol) 16:233-237, 2004

25. Paoletti AM, Congia S, Lello S, Tedde D, Orrù M, Pistis M, et al: Low androgenization index in elderly women and elderly men with Alzheimer's disease. Neurology 62:301-303, 2004

26. Peace KA, Orme SM, Padayatty SJ, Godfrey HPD, Belchetz PE: Cognitive dysfunction in patients with pituitary tumour who have been treated with transfrontal or transsphenoidal surgery or medication. Clin Endocrinol (Oxf) 49:391-396, 1998

27. Peace KA, Orme SM, Thompson AR, Padayatty S, Ellis AW, Belchetz PE: Cognitive dysfunction in patients treated for pituitary tumours. J Clin Exp Neuropsychol 19:1-6, 1997

28. Psaras T, Milian M, Hattermann V, Gerlach C, Honegger J: Executive functions recover earlier than episodic memory after microsurgical transsphenoidal resection of pituitary tumors in adult patients - a longitudinal study. J Clin Neurosci 18:1340-1345, 2011

29. Ragnarsson O, Stomby A, Dahlqvist P, Evang JA, Ryberg M, Olsson T, et al: Decreased prefrontal functional brain response during memory testing in women with Cushing's syndrome in remission. Psychoneuroendocrinology 82:117125,2017

30. Sangün Ö, Demirci S, Dündar N, Pirgon Ö, Koca T, Doğan M, et al: The effects of six-month L-thyroxine treatment on cognitive functions and event-related brain potentials in children with subclinical hypothyroidism. J Clin Res Pediatr Endocrinol 7:102-108, 2015

31. Takayanagi Y, Spira AP, McIntyre RS, Eaton WW: Sex hor- mone binding globulin and verbal memory in older men. Am J Geriatr Psychiatry 23:253-260, 2015

32. Tiemensma J, Kokshoorn NE, Biermasz NR, Keijser BJ, Wassenaar MJE, Middelkoop HAM, et al: Subtle cognitive impairments in patients with long-term cure of Cushing's disease. J Clin Endocrinol Metab 95:2699-2714, 2010

33. Tooze A: The Neurocognitive Consequences of Non-Functioning Pituitary Adenoma and Its Treatment [thesis]. Birmingham, UK: University of Birmingham, 2010

34. Tooze A, Hiles CL, Sheehan JP: Neurocognitive changes in pituitary adenoma patients after gamma knife radiosurgery: a preliminary study. World Neurosurg 78:122-128, 2012

35. Wang X, Tong X, Zou Y, Tian X, Mao Z, Sun Z: The impact on cognitive functions of patients with pituitary adenoma before and after surgery. Neurol Sci 38:1315-1321, 2017

36. Wechsler D: Wechsler Adult Intelligence Scale: Technical and Interpretive Manual, ed 4. San Antonio: Pearson, 2008

37. Yaffe K, Lui LY, Zmuda J, Cauley J: Sex hormones and cognitive function in older men. J Am Geriatr Soc 50:707-712, 2002

38. Yang CJ, Huang GS, Xiao FR, Lou MF: Symptom distress and quality of life after stereotactic radiosurgery in patients with pituitary tumors: a questionnaire survey. PLoS One 9:e88460, 2014

39. Zhu DF, Wang ZX, Zhang DR, Pan ZL, He S, Hu XP, et al: fMRI revealed neural substrate for reversible working memory dysfunction in subclinical hypothyroidism. Brain 129:2923-2930, 2006

\section{Disclosures}

This work was financially supported by an Elekta grant.

\section{Author Contributions}

Conception and design: both authors. Analysis and interpretation of data: Tooze. Drafting the article: Tooze. Critically revising the article: Sheehan. Reviewed submitted version of manuscript: both authors. Statistical analysis: Tooze. Study supervision: Sheehan.

\section{Supplemental Information \\ Previous Presentations}

This work was presented at the 19th Leksell Gamma Knife Society Meeting held in Dubai, United Arab Emirates, on March 4-8, 2018.

\section{Correspondence}

Jason P. Sheehan: University of Virginia, Charlottesville, VA. jsheehan@virginia.edu. 